\title{
Síntesis y uso de histidinato de cobre en niños con enfermedad de Menkes en México
}

\author{
Andrey Arturo Flores-Pulido, ${ }^{1}$ Víctor Manuel Jiménez-Pérez ${ }^{2}$ y Néstor Rodolfo García-Chong ${ }^{1}$ \\ ${ }^{1}$ Secretaría de Salud, Centro Regional de Alta Especialidad de Chiapas, Hospital de Especialidades Pediátricas, Chiapas; ${ }^{2}$ Universidad Autónoma \\ de Nuevo León, Facultad de Ciencias Químicas, Departamento de Materiales, Nuevo León, México
}

\begin{abstract}
Resumen
La enfermedad de Menkes es una patología neurodegenerativa y letal debida a mutaciones génicas de la enzima ATP-7A trasportadora de cobre; se manifiesta por síntomas neurológicos y alteraciones del tejido conectivo de severidad variable. El uso subcutáneo oportuno de histidinato de cobre (Cu-His) es determinante en la calidad de vida. Se reportan las primeras experiencias en México en la síntesis y uso seguro de Cu-His en tres casos en los que corroboramos hipocupremia e hipoceruloplasminemia. Bajo asesoramiento del Hospital for Sick Children, Toronto, Canadá, elaboramos una solución de $500 \mathrm{\mu g} / \mathrm{mL}$. En los tres casos aplicamos $250 \mu \mathrm{g}$ de Cu-His, sin efectos indeseables relevantes durante 30 días y observamos las siguientes determinaciones séricas de cobre ( $\mathrm{Cu}$ en $\mu \mathrm{g} / \mathrm{L}$ ) y ceruloplasmina ( $\mathrm{Cp}$ en $\mathrm{mg} / \mathrm{dL}$ ): caso 1, Cu días 0 y 30, 8 y $504 \mu \mathrm{g} / \mathrm{L}$; Cp días 0 y 30, 4 y 10.75 mg/dL; caso 2, Cu días 0 y 30, < 50 y 502, $\mu \mathrm{g} / \mathrm{L}$; Cp días 0 y 30, 2 y $15 \mathrm{mg} / \mathrm{dL}$; caso 3, Cu días 0 y 30, 3 y $84.2 \mu \mathrm{g} / \mathrm{L}$; Cp días 0 y 30, 4 y $10.7 \mathrm{mg} / \mathrm{dL}$. En México es posible la síntesis segura de Cu-His y tratar la enfermedad de Menkes, la cual debe ser intencionalmente buscada.
\end{abstract}

PALABRAS CLAVE: Menkes. Histidinato. Cobre. Niños.

\begin{abstract}
Menkes disease is a neurodegenerative and lethal pathology caused by gene mutations of the copper-transporting ATP-7A enzyme; it manifests itself by neurological symptoms and connective tissue changes of varying severity. Timely subcutaneous use of copper histidinate (Cu-His) is determinant for quality of life. We report the first experiences in Mexico on Cu-His synthesis and its safe use in 3 cases where hypocupremia and hypoceruloplasminemia were corroborated. With advice of the Hospital for Sick Children of Toronto Canada, we prepared a $500 \mu \mathrm{g} / \mathrm{mL}$ solution. In all three cases were $250 \mu \mathrm{g}$ of Cu-His applied without relevant undesirable effects for 30 days. Serum copper ( $\mathrm{Cu}$, expressed in $\mu \mathrm{g} / \mathrm{L})$ and ceruloplasmin (Cp, in $\mathrm{mg} / \mathrm{dL}$ ) were determined: case 1, Cu days 0 and 30, 8 and $504 \mu \mathrm{g} / \mathrm{L}$; Cp days 0 and 30, 4 and $10.75 \mathrm{mg} / \mathrm{dL}$; case 2, Cu days 0 and 30, <50 and $502 \mu \mathrm{g} / \mathrm{L}$; Cp days 0 and 30, 2 and $15 \mathrm{mg} / \mathrm{dL}$; case 3, Cu days 0 and 30, 3 and $84.2 \mu \mathrm{g} / \mathrm{L} ; \mathrm{Cp}$ days 0 and 30, 4 and $10.7 \mathrm{mg} / \mathrm{dL}$. In Mexico, it is possible to safely synthesize Cu-His and treat MD, which must be intentionally sought.
\end{abstract}

KEY WORDS: Menkes. Histidinate. Copper. Children.

\section{Introducción}

La enfermedad de Menkes, código OMIM\#309400, es una noxa recesiva ligada a $X$ poco frecuente (1 entre 35000 a 250000 nacidos vivos), pero tempranamente letal y neurodegenerativa; ${ }^{1,2}$ se debe a mutaciones interagénicas 0 deleciones en el gen codificante para la enzima ATP7A trasportadora de cobre, que afectan múltiples sistemas enzimáticos (Tabla 1$)^{3,4}$ y se manifiestan en un espectro clínico que varía entre la forma severa (más común) 0 Menkes clásico y el síndrome de cuerno occipital
Correspondencia:

Andrey Arturo Flores-Pulido

E-mail: andreyfp77@gmail.com
Fecha de recepción: 26-03-2018

Fecha de aceptación: 22-11-2018

DOI:10.24875/GMM.18004310
Gac Med Mex. 2019;155:191-195

Disponible en PubMed www.gacetamedicademexico.com 
Gaceta Médica de México. 2019;155

Tabla 1. Enzimas cobredependientes en mamíferos y su relación probable con la enfermedad de Menkes

\begin{tabular}{|c|c|c|}
\hline Enzima & Actividad biológica & Síntoma \\
\hline Oxidasa del citocromo $\mathrm{C}$ & Respiración celular & $\begin{array}{l}\text { Degeneración del sistema nervioso central, ataxia, debilidad muscular, falla } \\
\text { respiratoria }\end{array}$ \\
\hline Dismutasa de superóxido & Barrido de radicales libres & Degeneración del sistema nervioso central \\
\hline Ceruloplasmina & Trasportador de hierro y cobre & Anemia \\
\hline Hepaestina & Trasporte de hierro & Anemia \\
\hline Tirosinasa & $\begin{array}{l}\text { Formación de pigmentos } \\
\text { tegumentarios }\end{array}$ & Hipomelanosis \\
\hline Beta OH-asa de dopamina & Producción de catecolaminas & Ataxia, hipotermia, hipotensión, diarrea \\
\hline Peptidil alfa amidante & $\begin{array}{l}\text { Activación de péptidos } \\
\text { hormonales }\end{array}$ & Inespecíficos \\
\hline Lisil oxidasa & $\begin{array}{l}\text { Enlaces cruzados del colágeno } \\
\text { y la elastina }\end{array}$ & $\begin{array}{l}\text { Ruptura prematura de membranas, cefalohematoma, facies anormal, paladar } \\
\text { ojival, enfisema, hernias, divertículos vesicales, aneurismas arteriales, } \\
\text { hiperlaxitud articular y cutánea, osteoporosis, petequias, heridas dehiscentes, } \\
\text { degeneración del sistema nervioso central }\end{array}$ \\
\hline Sulfidrilo oxidasa & Cruzamientos de la queratina & Cabello anormal y piel seca \\
\hline
\end{tabular}

(OHS), con manifestaciones como retraso psicomotor severo, encefalopatía epiléptica, emaciación, falla de medro, hipopigmentación tegumentaria, tricodistrofia (pili torti), urocistodistrofia, osteoporosis, con supervivencia de los casos severos generalmente no más allá del tercer año de vida. ${ }^{3,5}$ Para el tratamiento y paliación se utiliza histidinato de cobre (Cu-His) como intervención de impacto clínico posiblemente relevante en el sistema nervioso central y en la calidad de vida, a tal punto que en pacientes que lo recibieron precozmente ha alargado la supervivencia a más de 30 años de vida en buenas condiciones clínicas. ${ }^{6-8}$ Reportamos las primeras experiencias en México en la síntesis de $\mathrm{Cu}$-His y los hallazgos con su uso en tres casos.

\section{Método}

Todos los reactivos fueron producidos por Sigma-Aldrich. La síntesis del Cu-His se llevó a cabo usando las instrucciones del Hospital for Sick Children de Toronto Canadá (versión de agosto de 2013, Deparment of Pharmacy-Compounding). El producto final derivado del cobre expira después de 56 días de ser sintetizado.

La preparación magistral del Cu-His $\left(500 \mu \mathrm{g} / \mathrm{mL}^{-1}\right)$ se realizó de la siguiente forma:

- Preparación de la solución de hidróxido de sodio $0.2 \mathrm{~N}$ en solución inyectable de cloruro de sodio a $0.9 \%$. Con el equipo de protección adecuado (lentes y guantes), se pesaron $400 \mathrm{mg}$ de hidróxido de sodio en la balanza analítica. Al tratarse de pellets fue necesario un cálculo de ajuste (por ejemplo, $440 \mathrm{mg}$ qs para $56 \mathrm{~mL}$ ). Los pellets se colocaron en un vaso de precipitados estéril, en la cabina de flujo laminar. Se añadieron $40 \mathrm{~mL}$ de cloruro de sodio a $0.9 \%$ en solución inyectable para disolver; se procedió a agitar el preparado. Después se agregó qs para $50 \mathrm{~mL}$, con solución inyectable de cloruro de sodio a $0.9 \%$ en un matraz volumétrico. Los mililitros adicionales a los $50 \mathrm{~mL}$ se preparan aparte utilizando una jeringa y se incorporan el vaso de precipitados, donde se mezclan.

- Preparación de la solución de Cu-His. Con el equipo de protección adecuado (lentes y guantes), se pesaron el cloruro de cobre y la L-histidina en la balanza analítica. El frasco fue pesado y regresado rápidamente al desecador (el cloruro de cobre es higroscópico). Los dos polvos se colocaron en el vaso de precipitados y se disolvieron en aproximadamente $185 \mathrm{~mL}$ de solución inyectable de cloruro de sodio a $0.9 \%$; la preparación fue agitada suavemente solo para disolverlo (el producto es sensible al oxígeno). Después de estandarizar, el electrodo (entre $\mathrm{pH} 4$ y 7 ) fue sumergido varias veces en alcohol a $99 \%$ para limpiarlo; se procedió a enjuagarlo. Se ajustó la solución a pH de 7.38 a 7.40, utilizando la solución de hidróxido de sodio $0.2 \mathrm{~N}$; se determinó el $\mathrm{pH}$ de la solución. Se tomaron aproximadamente $14 \mathrm{~mL}$ más 10 a 
13 gotas (dependiendo de la potencia del $\mathrm{NaOH}$ se emplearon más o menos); después de cada adición se mezclaron bien. Continuamente se monitoreaba el $\mathrm{pH}$. Una vez cercano al pH indicado (6.9 a 7.0), se añadió de media a una gota y se agitó lentamente. Al llegar al pH indicado, los electrodos fueron limpiados con agua estéril y la solución fue transferida a un matraz volumétrico. El volumen fue ajustado a $200 \mathrm{~mL}$ con solución inyectable de cloruro de sodio a $0.9 \%$. Se midió de nuevo el pH para asegurar que continuaba en el rango (en ocasiones se pueden necesitar gotas extra de $\mathrm{NaOH}$ ). La solución se transfirió a un vaso de precipitados. Se tomó solución de cobre-histidina en una jeringa de $20 \mathrm{~mL}$ y se filtraron $3 \mathrm{~mL}$ de la solución utilizando un filtro de disco de 0.22 micrones en viales de 5 o $10 \mathrm{~mL}$.

Es importante considerar algunas especificaciones técnicas:

- Los matraces y vasos usados en la preparación de la solución de cobre-histidina deben estar cubiertos con aluminio porque el producto es muy sensible a la luz. La luz de la campana deberá estar apagada durante la preparación. Si el cloruro de cobre se torna azul/verde, es indispensable verificar con el farmacéutico antes de su uso.

- Una vez preparado el primer vial, debe ser enviado a medición del nivel de cobre.

- El producto deberá permanecer de color azul aqua. Si se obscurece a verdeazulado significa que se está degradando.

- Una vez terminado, el producto no deberá ser golpeado o agitado.

Para evitar degeneración por exposición fótica 0 térmica, se dispuso de viales de $2 \mathrm{~mL}$ tipo falcon con coloración ámbar y se trasportaron en base soporte para mantenerlos verticales en contenedores, cuya temperatura interna debía oscilar entre 4 a $10{ }^{\circ} \mathrm{C}$

Para su dispensación unitaria se sugiere cargar dentro del área de servicios farmacéuticos en los minutos previos a su administración, en habitación con luz tenue y temperatura regulada y campana de flujo laminar en jeringa de $1 \mathrm{~mL}$ con aguja $27 \mathrm{G} \times 13 \mathrm{~mm}$, verificando la coloración adecuada y protegiendo el dispositivo de inyección cuanto antes con plástico oscuro.

Para la administración se utilizó la región deltoidea de ambos brazos, dividida en nueve cuadrantes para ordenar su aplicación diaria (Figura 1). ${ }^{7}$ La inyección

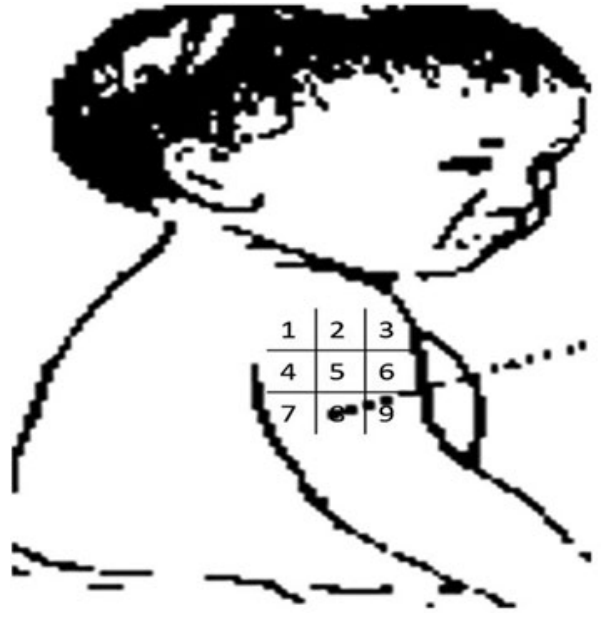

Figura 1. Cuadrantes de administración subcutánea del Cu-His en la región deltoidea (cara posterolateral del tercio proximal del brazo derecho e izquierdo). Fuente: Recreación de la imagen de la referencia 8.

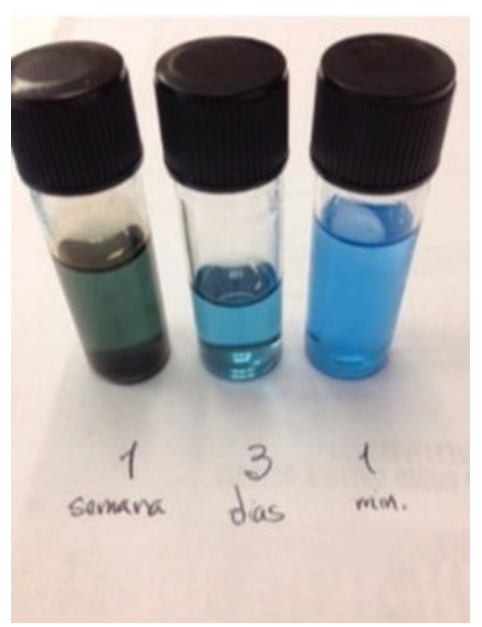

Figura 2. Apariencia de la solución. Fuente: Fotografía tomada en el laboratorio de materiales de la Facultad de Química de la Universidad Autónoma de Nuevo León con el primer lote de viales sintetizados.

debe ser vía subcutánea. La dosis estándar recomendada es de $250 \mu \mathrm{g} /$ dosis $(0.5 \mathrm{~mL})$ cada 24 horas. $^{2,3,4}$

\section{Resultados}

La solución resultante de la preparación -clara, azul aqua, fotosensible y termosensible (Figura 2) - fue aplicada sin efectos indeseables significativos relacionados con el sitio de administración subcutánea (deltoidea), ni sistémicos relevantes durante el seguimiento de los pacientes. Durante los primeros 30 días, la administración subcutánea diaria (previo consentimiento informado) de $250 \mu \mathrm{g}$ de $\mathrm{Cu}$-His en los pacientes portadores de enfermedad de Menkes genera cambios en las 


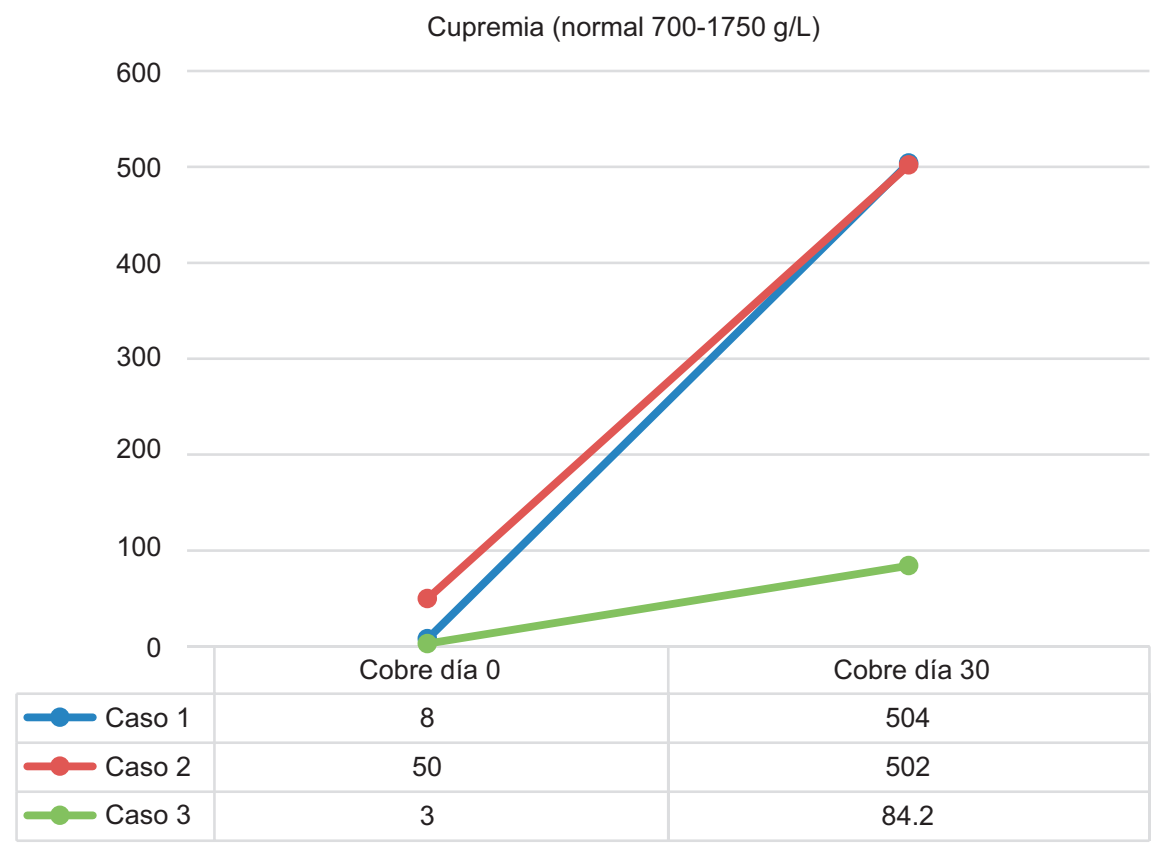

Figura 3. Cupremia resultante de 30 días de uso de Cu-His en los tres casos.

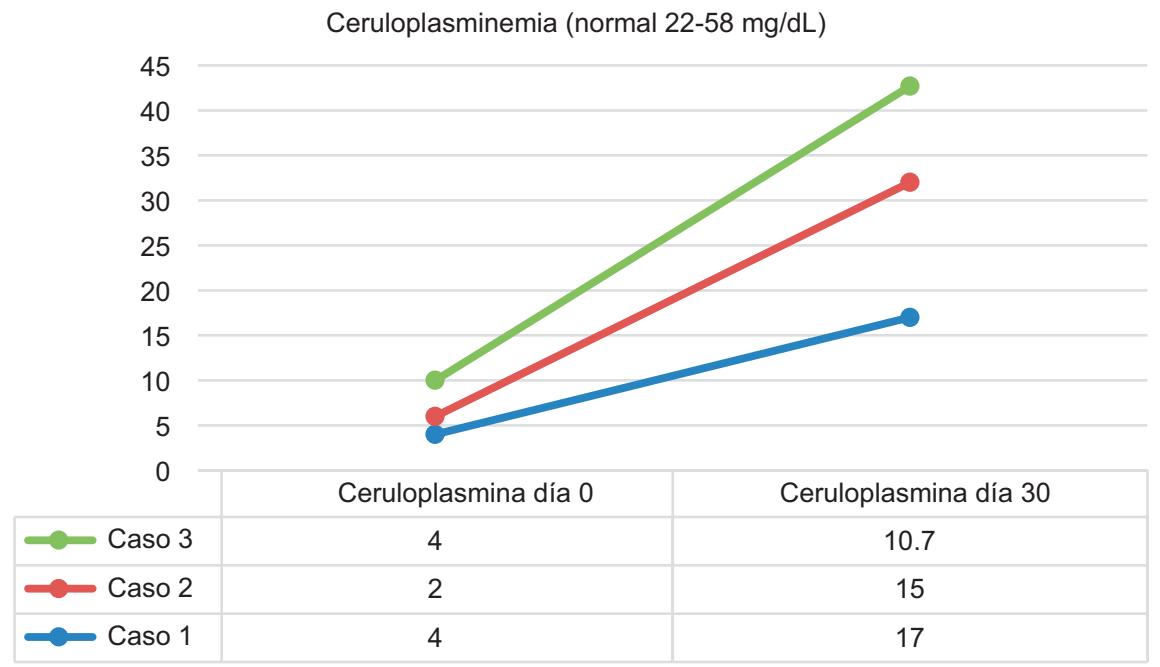

Figura 4. Ceruloplasminemia resultante de 30 días de uso de Cu-His en los tres casos.

determinaciones séricas de cobre $(\mathrm{Cu}$, nivel de referencia de 700 a $1750 \mu \mathrm{g} / \mathrm{L}$ ) y de ceruloplasmina (CP, nivel de referencia de 22 a $58 \mathrm{mg} / \mathrm{dL}$ ) (Figuras 3 y 4).

El caso 1 fue captado a los 12 meses de edad, el caso 2 a los 36 meses de edad y el caso 3 , a los cuatro meses de edad. En todos los casos se observó la atenuación del número y duración de los espasmos infantiles, así como mejoría en cuanto a la reactividad y alternancia del sueño-alerta. Solo el caso 3 presentó cambios tegumentarios, aun con niveles de $\mathrm{Cu}$ y $\mathrm{CP}$ inferiores a los de referencia. Al momento de este informe se continuaba con la medición espectroscópica y monitoreo del comportamiento de la $\beta$-microglobulina a manera de seguimiento de la función tubular.

\section{Discusión}

El Cu-His es una molécula endógena o sintética que refuerza la captura intracelular de cobre y a la vez es un modulador o amortiguador del intercambio de cobre entre la célula y la albúmina que regula su biodisponiblidad. ${ }^{2,9}$

En la enfermedad de Menkes, los niveles séricos de $\mathrm{Cu}$ y CP son ínfimos, primariamente por daño en la 
capacidad intestinal absortiva relacionada con la disfunción de la ATP7A, por lo que el tratamiento suplementario se enfoca en la restitución de este elemento. Recuperada la cupremia, se induce restitución de la ceruloplasminemia, lo que asegura su portación eficiente hacia las células, sin embargo, ya en el compartimiento plasmático debe evadirse la alta afinidad cúprica por la albúmina, lo que le resta biodisponiblidad, allí en donde radica la multitarea de moléculas como el $\mathrm{Cu}$-His, pues otras moléculas como el cloruro de cobre, sulfato de cobre, ácido etilendiaminotetraacético de cobre y la albúmina cúprica no han mostrado impacto clínico suficiente comparado con el $\mathrm{Cu}$-His. ${ }^{9-12}$

La recomendación es emplear $\mathrm{Cu}$-His para mejorar la biodisponiblidad de Cu en los sistemas enzimáticos cobredependientes a través de la vía subcutánea, evadiendo el defecto de la barrera enteral y facilitando y captando el transferencia de cobre desde la albúmina cúprica para mejorar su biodisponibilidad. Lo anterior se ha establecido conforme varios casos de detección temprana en el rango de cero a siete meses de edad, en los cuales el pronóstico de la evolución neurológica cambia drásticamente, sobre todo si se administra dentro de los primeros dos meses de vida. ${ }^{3,6,9-13}$ Nuestros casos tenían 12 y 36 y 4 meses de edad, con evolución de la enfermedad de Menkes clásica y cuyo principal problema era el estado nutricional y la epilepsia refractaria. Hay escasa información disponible acerca del inicio tardío de $\mathrm{Cu}$-His, que no puede contrastarse con el uso crónico de inicio temprano en la mayoría de las series reportadas (durante los primeros tres años de vida)., ${ }^{73}$

La decisión de desarrollar la síntesis en México - que no había sido posible dada la inestabilidad de esta sustancia en estado acuoso y de que no se había establecido la red logística que permitiera su disponibilidad segura - fue motivada por la posibilidad de modificar drásticamente la historia natural de los futuros casos y de disponer de una intervención tardía potencialmente benéfica, si bien de efecto incierto, que requiere vigilancia estrecha.

En el recuento de los obstáculos, la realización de pruebas de absorción atómica y la esterilidad constituyen los pasos procedimentales que más recursos y precisión demandan, a pesar de lo cual se realizaron de forma óptima.

A pesar del inicio tardío del Cu-His se observaron cambios plasmáticos y clínicos de mejora, por lo que se continuará su empleo con el monitoreo de hallazgos y su comparación con los de otras series.

Consideramos que en México es posible sintetizar colaborativamente y de forma segura el $\mathrm{Cu}$-His y acceder tempranamente al tratamiento de la enfermedad de Menkes, lo que implica una búsqueda intencionada y sistemática de casos en todo el sistema nacional, ya que muchos fallecen por la falta de detección oportuna.

\section{Agradecimientos}

Con el apoyo generoso de los doctores Sarkar y Walsh del Hospital for Sick Children, de Toronto, Canadá, y del doctor Víctor Manuel Jiménez de la Facultad de Ciencias Químicas, Universidad Autónoma de Nuevo León, ha sido posible sintetizar, asegurar, dispensar y administrar con seguridad el $\mathrm{Cu}$-His en el Centro Regional de Alta Especialidad de Chiapas, así como compartirlo remotamente gracias al apoyo logístico del laboratorio clínico, servicios farmacéuticos y dirección de operaciones. Trabajo en honor a la batalla que enfrentan Harold, Álan, Gabriel y sus familias en contra de la enfermedad de Menkes.

\section{Bibliografía}

1. Carbajal-Rodríguez L, Belmont-Martínez L, González-Del Ángel A, Delgado-Falcón J, Durán-C, Rodríguez-Herrera R, et al. Síndrome de Menkes. Bol Med Hosp Infan Mex. 2002;59:365-371.

2. Christodoulou J, Danks D, Sarkar B, Baerlocher K, Casey R, Horn N et al. Early treatment of Menkes disease with parenteral cooper-histidine: long term follow-up of four treated patients. Am J Med Genet. 1998;76:154-164.

3. Deschamps P, Kulkarni P, Gautam-Basak M, Sarkar B. The saga of copper (II)-L histidine. Coord Chem Rev. 2005;249:895-909.

4. Ojha R, Prasad AN. Menkes disease: what a multidisciplinary approach can do. J Multidiscip Healthc. 2016;9:371-385.

5. Garnica A, Chan WY, Rennert O. Copper-histidine treatment of Menkes disease. J Pediatr. 1994;125:336-338.

6. León-García, Dávila-Gutiérrez. Estado actual de la enfermedad de Menkes en México. Salud Publica Mex. 2012;54:103-104.

7. Menkes JH, Alter M, Steigleder GK, Weakley DR, Sung JH. A sex-linked recessive disorder with retardation of growth, peculiar hair, and focal cerebral and cerebellar degeneration. Pediatrics. 1962;29:761-779.

8. Vía de administración subcutánea en lactantes e infantes. Disponible en: https://image.slidesharecdn.com/1-tecnicasdeaplicacion-1302011 20246-phpapp01/95/1tcnicas-de-aplicacin-vacunas-13-638.jpg?$c b=1359720489$

9. Sarkar B, Lingertat-Walsh K, Clarke JTR. Cooper histidine therapy for Menkes disease. J Pediatr. 1993;123:828-830.

10. Tümer Z, Møller LB. Menkes disease. Eur J Hum Genet. 2010;18:511-518.

11. Tümer Z, Chirstodoulou J, Clarke JTR, Sarkar B, Horn N. Early cooper histidine treatment for Menkes disease. Nat Genet. 1996;12:11-13.

12. Kaler SG, Holmes CS, Goldstein DS, Tang J, Gosdwin S, Donsante A, et al. Neonatal diagnosis and treatment of Menkes disease. $\mathrm{N}$ Eng J Med. 2008;358:605-614.

13. Sheela SR, Latha M, Liu P, Lem K, Kaler SG. Copper-replacement treatment for symptomatic Menkes disease: ethical considerations. Clin Genet. 2005;68:278-283. 